\title{
SITUAÇÃO DO ENSINO DAS PRÁTICAS INTEGRATIVAS E COMPLEMENTARES NOS CURSOS DE GRADUAÇÃO EM ENFERMAGEM, FISIOTERAPIA E MEDICINA
}

Léia Fortes Salles', Rafael Fernandes Bel $\mathrm{Homo}^{2}$, Maria Júlia Paes da Silva ${ }^{3}$

1Enfermeira. Doutora em Ciências da Saúde. Universidade de São Paulo. São Paulo-SP-Brasil.

${ }^{2}$ Enfermeiro. Especialista em Auditoria em Enfermagem. Instituto do Câncer do Estado de São Paulo. São Paulo-SP-Brasil.

${ }^{3}$ Enfermeira. Doutora em Enfermagem. Universidade de São Paulo. São Paulo-SP-Brasil.

RESUMO: O objetivo foi analisar a situação do ensino das Práticas Integrativas e Complementares em faculdades de Enfermagem, Medicina e Fisioterapia no Brasil. A amostra foi constituída de faculdades públicas e a busca de dados ocorreu nos sites das instituições investigadas entre maio de 2012 a março de 2013. Das 209 instituições públicas de ensino superior, somente 43 (32,3\%) oferecem disciplinas relacionadas com o tema. Dentre os três cursos, as escolas de Enfermagem oferecem mais disciplinas, seguidas pela Medicina e Fisioterapia; 26,4\%, $17,5 \%$ e 14,6\%, respectivamente. A média da carga horária é de 46 horas e a maioria delas são oferecidas de forma optativa, ficando boa parte dos profissionais de saúde sem nenhuma aproximação acadêmica com esses saberes. As instituições de ensino, que formam profissionais para o mercado de trabalho e para o Sistema Único de Saúde, precisam rever as grades curriculares.

DESCRITORES: Terapias complementares; Ensino; Enfermagem; Medicina; Fisioterapia.

\section{THE SITUATION OF THE TEACHING OF HOLISTIC AND COMPLEMENTARY PRACTICES IN UNDERGRADUATE COURSESINNURSING, PHYSIOTHERAPYANDMEDICINE}

ABSTRACT: This study aimed to analyze the situation of the teaching of Integrative and Complementary Practices in faculties of Nursing, Medicine and Physiotherapy in Brazil. The sample was constituted of public faculties and the data search occurred on the websites of the institutions investigated, between May 2012 and March 2013. Of the 209 public higher education institutions, only $43(32.3 \%)$ offered courses related to this topic. Among these three courses, the schools of Nursing offer the most courses, followed by Medicine and Physiotherapy; $26.4 \%$, $17.5 \%$ and $14.6 \%$, respectively. The mean course load is 46 hours, and the majority are offered as options, with a large proportion of health professionals not studying these knowledges. The teaching institutions, which train professionals for the job market and for the Unified Health System, need to review the curriculum frameworks.

DESCRIPTORS: Complementary therapies; Teaching; Nursing; Medicine; Physiotherapy.

\section{SITUACIÓNDELENSEÑANZADELASPRÁCTICASINTEGRA- TIVASYCOMPLEMENTARIASENLOSCURSOSDEGRADU- ACIÓN EN ENFERMERÍA, FISIOTERAPIA Y MEDICINA}

RESUMEN: Fue objetivo del estudio analizar la situación de la enseñanza de las Prácticas Integrativas y Complementarias en facultades de Enfermería, Medicina y Fisioterapia en el Brasil. La muestra fue constituida de facultades públicas y la búsqueda de datos ocurrió en los sites de las instituciones investigadas entre mayo de 2012 y marzo de 2013. De las 209 instituciones públicas de enseñanza superior, solamente 43 (32,3\%) ofrecen asignaturas acerca del tema. Entre los tres cursos, las escuelas de Enfermería ofrecen más asignaturas, seguidas por la Medicina y Fisioterapia; $26,4 \%$, $17,5 \%$ y $14,6 \%$, respectivamente. La media de horas es de 46 y la mayoría de ellas son ofrecidas de forma optativa, quedándose una grande parte de los profesionales de salud sin aproximación académica con eses saberes. Las instituciones de enseñanza, que forman profesionales para el mercado de trabajo y para el Sistema Único de Salud, necesitan analizar los currículos.

DESCRIPTORES: Terapias complementarias; Enseñanza; Enfermería; Medicina; Fisioterapia. 


\section{INTRODUÇÃO}

As Práticas Integrativas e Complementares em Saúde (PICS) foram reconhecidas no Brasil em 2006 e deveriam ser oferecidas amplamente no Sistema Único de Saúde ${ }^{(1)}$.

Sob a classificação das PICS estão diversas técnicas com finalidades de promoção à saúde, prevenção e tratamento de doenças, que embora reconhecidas pelo uso popular desde a antiguidade, ainda não fazem parte da maioria dos programas oficiais da saúde, principalmente nos países ocidentais ${ }^{(2)}$. Entre outras formas de cuidado com a saúde, podemos citar a acupuntura, antroposofia, aromaterapia, auriculoterapia, fitoterapia, hidroterapia, iridologia, massagens terapêuticas, meditação, musicoterapia, quiropatia, reiki, reflexologia, relaxamento, terapia floral e toque terapêutico(2).

Um complexo conjunto de processos globais vem trazendo de volta o uso dessas práticas. Dentre eles podemos citar as amplas mudanças culturais como o aumento da expectativa de vida, das doenças crônicas e degenerativas e nos custos dos cuidados de saúde, maior acesso à informação por meio da internet e novos movimentos sociais ligados à saúde ${ }^{(3)}$.

Um dos desafios mundiais de saúde é encontrar novas formas de prevenir o aparecimento de doenças crônico-degenerativas ${ }^{(3)}$. As PICS podem ser uma ferramenta útil para o enfrentamento deste desafio na medida em que auxiliam na manutenção da homeostase ao longo da vida e, nas idades mais avançadas, podem melhorar a saúde, aumentar o bem-estar e a capacidade funcional(2).

O aumento na procura pelos atendimentos com as PICS é fato global. Profissionais de saúde começam a estudar estas práticas, tanto para incluí-las nos seus repertórios de diagnóstico e tratamento como para pesquisá-las, investigando sua eficácia e segurança. Procuram também obter conhecimentos para poder discutir e/ou orientar os pacientes sobre as melhores escolhas, vantagens e desvantagens no seu uso ${ }^{(4)}$.

Na Alemanha, desde 2003, os currículos médicos têm uma carga horária destinada para as PICS(5). Na Espanha, em alguns estados como Barcelona e Zaragoza, são oferecidos mestrados em terapias naturistas para médicos e enfermeiros ${ }^{(6-7)}$.
Com o aumento das discussões e debates sobre as PICS, os profissionais que as utilizam têm o desafio de encontrar evidências de segurança e efetividade por meio de modelos de pesquisa "padrão ouro" mais adaptados à realidade próprias dessas práticas ${ }^{(2)}$. Nos últimos 10 anos houve um grande aumento no financiamento da investigação das PICS nos Estados Unidos, Canadá e Reino Unido, fortalecendo as pesquisas na área. No Reino Unido, por exemplo, fundações privadas, hospitais renomados e o Ministério da Saúde uniram-se em prol destas pesquisas. As fundações apoiaram financeiramente, hospitais abriram espaço para os estudos e o Ministério estabeleceu a regulamentação formal do processo de investigação, além de custear um programa de bolsas de doutorado e pós-doutorado nesta área. Isto permitiu um significativo processo de desenvolvimento acadêmico das PICS nas universidades britânicas ${ }^{(8)}$.

No Brasil, a Portaria No 971 de 3 maio de 2006, conforme já referido, aprova a Política Nacional de Práticas Integrativas e Complementares (PNPIC) no Sistema Único de Saúde-SUS ${ }^{(1)}$.

É possível identificar que o desenvolvimento desta política incorporada ao SUS é um aprofundamento do cuidado em saúde, em busca da integralidade da atenção, acesso a serviços e exercício da cidadania e compreender que várias práticas complementares têm sido desenvolvidas na rede pública estadual e municipal de saúde de diferentes estados brasileiros, de forma desigual e descontinuada devido à ausência de diretrizes específicas $^{(9)}$.

A integralidade do cuidado à saúde, estabelecida como diretriz pela $8^{\text {a }}$ Conferência Nacional de Saúde e formalizada como princípio doutrinário do Sistema Único de Saúde, aponta para a oferta dessas práticas no Brasil. Deste modo, o objetivo da Portaria $n^{\circ} 971$ é fomentar o incremento de abordagens em saúde e tornar disponíveis opções promocionais, preventivas e terapêuticas diversas aos usuários do SUS e inserção dessas práticas significa respeito aos princípios do sistema ${ }^{(10)}$.

Dentre as profissões brasileiras, a Enfermagem foi pioneira no reconhecimento das terapias complementares como prática que pode ser exercida por seus profissionais. O Conselho Federal de Enfermagem reconhece e estimula os enfermeiros interessados na área a estudarem a 
prática escolhida com a profundidade necessária para o conhecimento e desenvolvimento de habilidades a fim de proporcionar uma assistência integral ao paciente (Resolução COFEN 197/1997 $(19 / 01 / 1997)^{(11)}$.

Estudo realizado na Espanha revela que apenas $21,3 \%$ instituições públicas de ensino de Enfermagem existentes no país oferecem disciplinas relacionadas com as PICS. As autoras da pesquisa concluem que com esta lacuna no ensino, a assistência de Enfermagem sofre uma perda na medida em que estes profissionais não dispõem de informações para orientar os pacientes sobre os tratamentos disponíveis e que isso, inclusive, fere o código de ética da profissão $^{(12)}$.

A homeopatia e a acupuntura, especificamente, foram reconhecidas pelo Conselho Federal de Medicina em 1980 e 1995, respectivamente, e fazem parte das especialidades médicas oferecidas em diversas instituições, sendo reembolsadas pelas seguradoras de saúde ${ }^{(13)}$.

Quanto a Fisioterapia, seu Conselho reconhece as práticas integrativas e complementares de saúde desde $2010^{(14)}$.

Esta pesquisa teve o objetivo de analisar a situação do ensino dessas práticas em faculdades de Enfermagem, Medicina e Fisioterapia no Brasil, considerando a necessidade de profissionais capacitados para poder implementar a portaria citada.

Como justificativa e importância do estudo destaca-se o reconhecimento da real situação do ensino das PICS, reiterando que elas fazem parte da lista de serviços que a população tem o direito de receber. Nossa hipótese é que estas práticas não são oferecidas pela maioria das graduações de Enfermagem, Fisioterapia e Medicina.

\section{MATERIAIS E MÉTODOS}

Trata-se de um estudo descritivo, exploratório com abordagem quantitativa.

A amostra foi constituída de 209 faculdades públicas de Enfermagem, Medicina e Fisioterapia do Brasil, catalogadas por meio de pesquisa junto aos órgãos oficiais de Educação, como o Ministério da Educação, e os Conselhos de cada profissão.

Primeiramente, a investigação foi realizada na web de cada instituição, com a leitura do plano curricular e, quando não foram encontradas informações suficientes no site, foi enviado e-mail para a direção da instituição com as dúvidas. Finalmente, na ausência de resposta foi tentado um contato telefônico. Vale ressaltar que essas informações são de domínio público (qualquer docente, aluno ou visitante pode ter acesso a esses dados).

Destaca-se que somente disciplinas específicas das PICS ou similares foram consideradas. As técnicas inerentes à profissão, como é o caso da massagem, para a Fisioterapia e a massagem de conforto para a Enfermagem não foram incluídas.

Os dados coletados entre maio de 2012 a março de 2013 foram compilados em um banco de dados no software da Microsoft Excel 2007 e analisados por frequência e porcentagem.

\section{RESULTADOS}

Foram catalogadas 87 instituições públicas de ensino superior em Enfermagem, 74 em Medicina e 48 em fisioterapia.

A distribuição regional destes cursos de graduação oferecidos pela rede pública de ensino pode ser observada na Tabela 1.

Tabela 1 - Distribuição geográfica dos cursos da rede pública que oferecem disciplinas relacionadas ao PICS. São Paulo-SP-Brasil, 2013

\begin{tabular}{llllrlc}
\hline Regiões & \multicolumn{2}{c}{ Enfermagem } & \multicolumn{2}{c}{ Fisioterapia } & \multicolumn{2}{c}{ Medicina } \\
\hline Centro-oeste & 09 & $10,4 \%$ & 04 & $8,3 \%$ & 06 & $8,1 \%$ \\
\hline Sudeste & 25 & $28,7 \%$ & 15 & $31,3 \%$ & 22 & $29,7 \%$ \\
\hline Sul & 17 & $19,6 \%$ & 11 & $22,9 \%$ & 11 & $14,9 \%$ \\
\hline Nordeste & 26 & $29,8 \%$ & 14 & $29,2 \%$ & 25 & $33,8 \%$ \\
\hline Norte & 10 & $11,5 \%$ & 04 & $8,3 \%$ & 10 & $13,5 \%$ \\
\hline Total & 87 & $100 \%$ & 48 & $100 \%$ & 74 & $100 \%$ \\
\hline
\end{tabular}


Tabela 2 - Oferta de disciplina relacionada com PICS nas graduações de Enfermagem, Fisioterapia e Medicina. São Paulo-SP-Brasil, 2013

\begin{tabular}{llllllc}
\hline Cursos & \multicolumn{2}{c}{ Sim } & \multicolumn{2}{c}{ Não } & \multicolumn{2}{c}{ Perda dos dados } \\
\hline Enfermagem & 23 & $26,4 \%$ & 55 & $63,3 \%$ & 09 & $10,3 \%$ \\
\hline Fisioterapia & 07 & $14,6 \%$ & 37 & $77,1 \%$ & 04 & $8,3 \%$ \\
\hline Medicina & 13 & $17,5 \%$ & 53 & $71,7 \%$ & 08 & $10,8 \%$ \\
\hline
\end{tabular}

Dentre as 87 instituições públicas de ensino superior em Enfermagem, 23 (26,4\%) oferecem disciplinas relacionadas com essas práticas, $55(63,3$ \%) não oferecem e em nove (10,3\%) os dados não são conhecidos. Das 23 instituições que oferecem essa disciplina, oito (35\%) estão localizadas na região nordeste do país, em seis $(26,1 \%)$ elas tem caráter obrigatório e nas outras 17 (73,9 \%) são optativas e, a carga horária varia de 28 a 60 horas (média de 44 horas). Em 20 escolas a disciplina engloba a maioria das PICS e em três, somente fitoterapia.

Das 48 instituições públicas de ensino superior em Fisioterapia, sete $(14,6 \%)$ oferecem as disciplinas relacionadas com as PICS, 37 $(77,1 \%)$ não oferecem e em quatro $(8,3 \%)$ não se conhecem os dados. Dentre as disciplinas oferecidas, cinco $(71,4 \%)$ são optativas e duas $(28,6 \%)$ são obrigatórias; e três ficam na região nordeste. A carga horária varia de 30 a 68 horas (média de 49 horas). Dessas sete disciplinas, $42,8 \%$ são sobre acupuntura, $14,4 \%$ sobre arteterapia e 42,8\% sobre as diferentes práticas.

Dentre as 74 faculdades de Medicina, 13 $(17,5 \%)$ oferecem disciplinas relacionadas ao tema (6 com mais de uma disciplina), 53 (71,7\%) não oferecem e em oito $(10,8 \%)$ casos não se conhecem os dados. Em todas (100\%) as instituições, as disciplinas têm caráter de optativa e a carga horária variou entre 15 e 75 horas (média de 45 horas). Oito faculdades estão localizadas na região sudeste e a maioria das disciplinas é sobre acupuntura, homeopatia ou fitoterapia e, somente em três instituições a disciplina aparece com abordagem de diversas práticas.

Dentre as três graduações, as escolas de Enfermagem oferecem mais disciplinas com o tema seguidas pelas de Medicina e Fisioterapia; $26,4 \%, 17,5 \%$ e $14,6 \%$, respectivamente.

\section{DISCUSSÃO}

Os resultados apresentados revelam que ainda são poucas as instituições que oferecem disciplinas relacionadas com as PICS, embora elas façam parte da Política Nacional de Saúde e sejam reconhecidas integralmente pelo Conselho Federal de Enfermagem e Fisioterapia e, em parte, pelo Conselho Federal de Medicina. Estes números revelam a lacuna no conhecimento do profissional de saúde, que diminui sua área de atuação com as práticas propriamente, e também na orientação que deveria fazer ao seu paciente interessado em discutir e/ou utilizar tais práticas.

A "disputa" por tempo na grade curricular mínima obrigatória não é recente e, à medida que o conhecimento cresce, as instituições de ensino rediscutem "quantas horas" se usará para tal ou qual conteúdo. Inserir o ensino de práticas complementares de saúde na grade curricular dos profissionais de saúde é uma ação complexa e não tem solução simples. Há motivos corporativistas, econômicos, religiosos a se considerar quando se deseja aprofundar o entendimento desses outros saberes e quando se propõe analisar a perspectiva de mudança de paradigmas no campo de saúde. Pode-se afirmar que é uma mudança paradigmática quando se dá igual valor às dimensões emocionais, psicológicas, energéticas no cuidar/ tratar de um indivíduo, à dimensão física/bioquímica ${ }^{(15)}$.

É compreensível lembrar que todo grupo profissional necessita de regras que devem ser obedecidas coletivamente para a organização funcionar adequadamente. Como ensinar, portanto, sem "ameaçar" a estrutura do saber vigente, agregando valores, percepções, possibilidades de compreensão diagnósticas, é um aspecto que não pode ser esquecido para entender essa baixa inserção das práticas complementares em saúde ao ambiente acadêmico.

Ao propormos em uma disciplina, por exemplo, que a "energia" é um operador estratégico na configuração do corpo com uma significação simbólica central na organização da vida, estamos sugerindo que o paradigma energético não se reduz a um tema específico de física, mas que é parte da compreensão do mundo. Oferecer uma disciplina de práticas complementares no currículo 
pode significar, também, discutir modalidades terapêuticas ocidentais que entendem energia como um movimento biomagnético (reikianos e bioenergéticos) ou um circuito elétrico integrado (acupunturistas) ou como uma energia de cura

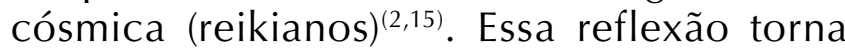
possível o questionamento do quanto interessa às instituições abrir esse leque de reflexão junto ao aluno que passará o restante de sua formação num único modelo.

Dentre os três cursos, a Enfermagem é a que oferece mais disciplinas ligadas ao assunto e de maneira mais ampla, com as diferentes práticas. Provavelmente este fato está relacionado ao pioneirismo do Conselho Federal de Enfermagem no reconhecimento das $\mathrm{PICS}^{(11)}$. Porém, com essa carga horária oferecida, e em apenas $1 / 4$ dos cursos de graduação, fica claro o quanto é preciso rever o ensino e formação básica desses futuros profissionais, se a intenção for "despertá-lo" para essa possibilidade de cuidado humano.

A graduação da Medicina privilegia os assuntos acupuntura e homeopatia, práticas já reconhecidas pelo seu Conselho Federal. Porém, a disciplina Medicina Integrativa começa a ser inserida mais sistematicamente em alguns currículos, seguindo a tendência mundial de uma abordagem holística para integrar o que há de melhor entre os tratamentos alopáticos, nutrição, atividades físicas e as práticas complementares para cada paciente.

Na graduação de Fisioterapia, assuntos como hidroterapia e massagem não foram considerados como disciplinas das PICS, uma vez que são temas inerentes da profissão. Das práticas ensinadas, a acupuntura tem destaque, embora desde 2010 o seu Conselho profissional reconheça as diferentes práticas complementares. Por ser este reconhecimento relativamente recente, pode-se justificar a sua não inserção no currículo.

A maioria das instituições que oferecem disciplina com as PICS situam-se no nordeste, uma das regiões mais carentes do país. Este achado encontra ressonância com as descrições da Organização Mundial da Saúde, a respeito da utilização destas práticas pelos países carentes do terceiro mundo ${ }^{(16)}$.

O quadro de ensino atual das três graduações aponta que o sucesso do ancoramento da Política Nacional das Práticas Integrativas e Complementares no SUS está diretamente ligado às possibilidades de articulação com a academia, que é responsável por prover profissionais no mercado de trabalho capazes de agregar outros saberes além do alopático, que sejam realmente mais populares (exemplo a fitoterapia), complementares e até, alternativos (ex: a homeopatia de linha unicista) ${ }^{(15)}$.

O cuidado profissional mais condizente e aproximado com o saber popular - que utiliza tais práticas só poderá se efetivar a partir do entendimento e do respeito sobre a diversidade cultural humana ${ }^{(1,17)}$. Este conhecimento, ainda que básico, permitirá ao profissional discutir vantagens e desvantagens com os pacientes que queiram utilizá-las ou ainda para os já fazem uso, muitas vezes de forma inadequada, como é o caso do uso de fitoterápicos ${ }^{(18)}$.

\section{CONCLUSÃO}

Embora as PICS sejam reconhecidas no sistema de saúde público brasileiro e pelos Conselhos Federais de Enfermagem e de Fisioterapia e, em parte, pelo Conselho Federal de Medicina, ainda são poucas as instituições que oferecem disciplinas relacionadas. As poucas que oferecem, o fazem de maneira optativa, ou seja, boa parte dos profissionais de saúde está se formando sem nenhuma aproximação acadêmica com seu estado de arte.

Dentre os três cursos, as escolas de Enfermagem oferecem mais disciplinas com o tema seguidas pelas de Medicina e Fisioterapia. A média de carga horária é de 46 horas nos três cursos.

Esta lacuna no conhecimento, no mínimo, impede o profissional de considerar todas as possibilidades de tratamento e cuidado para a assistência integral ao paciente, o que pode configurar um grave problema, inclusive, ético na saúde. Urge repensar essa problemática nas instituições de ensino.

Há, sim, uma emergência irrecusável, apesar de sua diversidade, de um novo repensar sobre o complexo campo de ensino na área da saúde, pois o resultado do uso dessas práticas tem impacto mundial inegável e algumas universidades defendem - na sua essência - o valor da pluralidade científica. Mesmo que tal pluralidade aponte para novas técnicas, saberes, diagnósticos que se confrontam com o paradigma biomédico vigente. 


\section{REFERÊNCIAS}

1. Ministério da Saúde (BR). Portaria n. 971, de 03 de maio de 2006. Dispõe sobre a Política Nacional de Práticas Integrativas e Complementares no Sistema Único de Saúde. Diário Oficial da União, Brasília (DF), 4 maio 2006. Seção 1, p. 20.

2. Salles LF, Silva MJP (organizadores). Enfermagem e as Práticas Complementares em Saúde. São Caetano do Sul (SP): Yendis; 2011.

3. Mendes EV. O cuidado das condições crônicas na atenção primária a saúde. O imperativo da consolidação da estratégia da saúde da família. [Internet]. Organização Pan Americana de Saúde - Brasília; 2012. [acesso em 10 dez 2013]. Disponível: http://apsredes.org/ site2012/wpcontent/uploads/downloads/2012/04/ RedesdeAtencaocondicoescronicas.pdf

4. Christensen MC, Barros NF. Complementary and alternative medicine in medical teaching: systematic review. Rev. bras. educ. med. 2010;34(1):97- 105.

5. Münstedt K, Harren $H$, von Georgi $R$, Hackethal A. Complementary and Alternative Medicine: Comparison of Current Knowledge, Attitudes and Interest among German Medical Students and Doctors. Evid Based Complement Alternat Med. [Internet]. 2011;2011. [acesso em 10 dez 2013]. Disponível: http:// www.ncbi.nlm.nih.gov/pubmed/19098296

6. Col legi Oficial Infermeres \& Infermers Barcelona - COIB. Màster en Medicina Naturista I Infermeria Naturista. Barcelona: Col legi Oficial Infermeres \& Infermers Barcelona [Internet] [acesso em 29 mar 2012]. Disponível: http://www.coib.cat/Generiques. aspx?idpagina $=8662$

7. Medicina Naturista. Curso de postgrado de Medicina Naturista de la Universidad de Zaragoza [Internet]. Zaragoza: Medicina Naturista de la Universidad de Zaragoza; 2012 [acesso em 28 mar 2012]. Disponível: http://www.medicinanaturista.org/content/view/21/6/

8. Lewith G, Verhoef M, Koithan M, Zick SM. Developing CAM Research Capacity for Complementary Medicine. Advance Access Publication. 2006;3(2):283-9.

9. Barros NF, Siegel P, Simoni S. Política Nacional de Práticas Integrativas e Complementares no SUS: passos para o pluralismo na saúde. Cad. Saúde Pública. [Internet] 2007;23(12) [acesso em 27 nov 13]. Disponível: http://dx.doi.org/10.1590/S0102311X2007001200030

10. Thiago SCS, Thesser CD. Terapias complementares: percepção de profissionais ESF. Rev Saúde Pública. 2011;45(2):249-57.

11. Conselho Federal de Enfermagem - COFEN. Resolução
COFEN 197/1997 de 19 de março de 1997 [Internet]. Brasília: Conselho Federal de Enfermagem; 1997 [acesso em 02 abr 2012]. Disponível: http://site. portalcofen.gov.br/node/4253

12. Cervilla ABF, Vives MEC, Dorado AIP. Situación actual de las terapias complementarias en España en el grado de enfermería. Rev. Latino-Am. Enfermagem. 2013;21(3):679-86.

13. Conselho Federal de Medicina - CRM. Resolução CFM 1785/2006 [Internet]. Brasília: Conselho Federal de Medicina; 2006 [acesso em 02 abr 2012]. Disponível: www.portalmedico.org.br/resolucoes/ cfm/2006/1785_2006.htm

14. Conselho Federal de Fisioterapia e Terapia Ocupacional - COFITO. Resolução n. 308 de 3 de novembro de 2010. [Internet]. Brasília: Conselho Federal de Fisioterapia e Terapia Ocupacional; 2010 [acesso em 02 abr 2012]. Disponível:http://www.coffito.org.br/ publicacoes/pub_viewasp?cod=1934\&psecao=9

15. Martins $\mathrm{PH}$. As outras medicinas e o paradigma energético. In: Luz MT, Barros NF (organizadores). Racionalidades médicas e práticas integrativas em saúde - estudos teóricos e empíricos. Rio de Janeiro: UERJ / IMS /LAPACIS; 2012. p. 309-41.

16. World Health Organization. WHO Traditional Medicine Strategy 2002-2005. Geneva: WHO; 2002.

17. Budó MLD, Resta DG, Denardin JM, Ressel LB, Borges ZN . Práticas de cuidado em relação à dor - a cultura e as alternativas populares. Esc. Anna Nery. 2008;12(1):90-6.

18. Reiner AAO, Ricci HA, Azevedo RCS. Uso de plantas medicinais para tratar a malaria. Cogitare enferm. [Internet]. 2010;15(3):454-9 [acesso em $10 \mathrm{dez} 2013]$. Disponível: http://ojs.c3sl.ufpr.br/ojs2/index.php/ cogitare/article/view/18887/12196 